\title{
Articulation Practices in Designing Teacher Training Courses: Perspectives and Differentiations in Complicated, Rephrased Patterns of Truth and Functionality
}

\author{
Kalerante Evaggelia \\ University of Western Macedonia \\ Florina, Greece
}

\begin{abstract}
This paper delves into the process of developing and providing educational training courses to teachers. In this respect, issues of adaptive policies are analysed based on the underlying system necessities. The selective correlations conducive to structuring converging fields among the social, political and cultural domains are also defined. Developmental operations about the sub-sampling of training models are clarified in such a way so as to highlight an educational policy on teachers' training in the form of a symbolic universe characterised by the educational domain interdependence and confined autonomy. The paper focuses on the constructed "status of truth" to meet teachers' needs. An obsessive quest for degrees and certification is prevailing, while teachers' personal time is being shrunk by the imposed "demands". The issue of domineering educational training packages is emphasized, as they cannot be considered educational discourse regardless of their technical or pedagogical features.
\end{abstract}

Keywords: educational packages; knowledge; lifelong education; teachers; training courses.

\section{Introduction}

The issue of teachers' training derives from the necessity for lifelong education. Shaping of educational discourse is gradually being developed towards determining cognitive settings while modern discourse in education is being formulated. The interest of education policy is seemingly shifted from university studies to alternative education and training. In this respect, a number of public and private institutes are involved in designing training courses. Moreover, they illustrate different conceptualisations about teachers' education, needs and approaches. 
An expanded teacher audience attends various training courses, as they essentially try to fulfil needs dictated and predetermined by the system (Harvey, 2007). Based on this arbitrary mapping of training courses, the interest in lifelong education is shifted from knowledge to formal participation and obtaining of a supplementary degree - certification (Belfield \& Levin, 2007; Kalerante, 2015; McMinn, 2015). The on-going number of training institutes involved in training courses is conducive to confining people in participating in processes which do not put forward knowledge. They neither disseminate scientific knowledge, nor operate as means of self-satisfaction and understanding the dimensions of the economic, social, political and cultural fields. Emphasis is obviously placed on developing phases, particularly tied to the "consumption" of training courses. Advancement or development of the teachers' career is tightly interweaved with on-going participation in further training, with lacking interest in expanding the content of knowledge, in interpretations and educational patterns. Values, needs and goals are reciprocally determined resulting in the training courses' "misleading picture" about the role of education and teachers' action. The single-sided process of consuming a pre-constructed truth about training courses perhaps makes the educational community inert. At the same time, the prevailing quest for certifications and degrees, imposed by the labour market "demands", is conducive to shrinking teachers' personal time.

The present paper seeks to analyse issues tied to delivering training courses, emphasizing the understanding and interpretation of this phenomenon. The generic field of education, educational community and educational normative implementations is being outlined within a range of particular economic, social, political and cultural views. Exploring training courses and depicting our theoretical approaches does not mean the silencing or refuting of the value pertaining to courses distinctive of their multi-range scientific and methodological approaches, as they contribute to developing scientific thought on education and understanding educational issues.

As it will be stated in the concluding remarks, a selective formation of training courses is proposed aiming at reinforcing critical attitude and associating knowledge with the educational process within a scientific view of complicated educational issues. The necessity to design and organize these courses is consequently not questioned. However, a number of educational training packages are inappropriate, as irresponsible carriers create poorly designed and scientifically unjustified forms of educational "eclecticism".

\section{Training courses in lifelong education}

Lifelong education is tied to lifelong involvement in knowledge whose content is re-formulated within the symbolic setting of constructing meanings in economic, social and political terms. Knowledge becomes symbolic and it is reflected in the form of complex narrative, unfolded in dynamic differentiated fields. Competitions, alterations, stabilisations and destabilisations are all conducive to constructing and reconstructing knowledge. Teachers' implemented training, 
explicitly or implicitly, showcases convergence with interpreted educational needs as well as with broader system demands (Sengupta \& Blessinger, 2018).

Obviously, offering educational training courses by hybrid private educational institutes (Kalerante, 2018) is gradually being expanded. These institutes are part of the knowledge market, as they take advantage of the vulnerability to design complete educational training courses offered by institutionalised public carriers. Legalised certifications assisting professional advancement have been indirectly conducive to symbolic constructions carried out by private institutions. Being confined and isolated within a fluid setting of refuted rights and intense uncertainty, teachers try to expand their formal qualifications so that they avoid exclusion and marginalization on the one hand, while reinforcing choices of professional advancement on the other. In this respect, a number of teachers attend seminars in order to claim a job position in the labour market, while others attend seminars simply to improve their current job position.

Lifelong education has also been included in the adaptive operational demands system through establishing a new market of training courses. Educational codes, scientific discourse and educational centres are all interconnected under a process of knowledge construction conceptualized, at the same time, within the disoriented model of educational culture. These processes are not systematically interactive. On the contrary, there is a passive attitude towards these training courses which are "consumed" (Graeber, 2018) by teachers. Their choice to attend them is seemingly proof of the educational content. However, they actually attend them rather typically and participate rather apathetically, being in a semi-conscious stage of involvement in a quest of vague educational opportunities.

Normative reference to the imperative nature of training is enough to legalize these courses. Training, allocating time and obtaining certificates become an end in itself regardless of potential content evaluation. The on-going number of available educational courses by different institutes and content-related misunderstanding are the outcome of any inner-educational circumscriptions that should be set by the educational centre bodies in charge (Makrakis, 1997). In this respect, these private centres gradually become autonomous entities, while putting forward the argument for independent education, as post-policy educational practice and uncommitted educational discourse.

Independent education is theoretically conceptualized by private institutes on the grounds of modernisation, leading up to broader education-related redistributions. Therefore, re-examining the structure and content of teachers' training courses is of primary importance. This can be done through broader exploration of this educational phenomenon emphasizing its conceptual framework and the general formulated tendency towards private education. In other words, the production and reproduction of knowledge as well as the complex relations among knowledge, educational implementations and interests can be re-evaluated through studying these training courses. The issue of training courses has not obviously been re-evaluated to date in the sense that they have been considered an essentially fragmented and harmless education 
system (Zervas, n.d.) whose structures and carriers were represented individually, mainly based on their business role ${ }^{1}$.

This expanded phenomenon and the gradual understanding that all these theoretically independent private institutes are included in the educational setting of knowledge management should motivate the educational unionist carriers $^{2}$ and the corresponding political ones. It is essential that these institutes be eventually evaluated upon certain criteria which will be the outcome of broader scientific and political estimations. The necessity for evaluation is based on the realisation that a form of exemplary manipulation of education is being unfolded by hybrid educational centres involved in monitoring lifelong education structures.

The increased number of educational packages offered by various institutes is obviously tied to the economic game. They are produced, delivered and finally purchased in the education market, not due to their content, but rather due to their symbolic meaning developed by the structural necessity to obtain a certification. Thus, developing and purchasing educational packages is based on an inventive justification about their necessity, prominent within the system of educational symbolic interactions.

The educational package of knowledge is gradually tied to a differentiated narration about their function in the institutional setting of people's advancement or the symbolic interaction among people participating in training which is eventually nothing more than a misleading process. Perhaps, the contact with actual knowledge has already been refuted at University, as knowledge acquisition is considered a utopia lost in the dense narrative about the necessity of piecemeal functional knowledge capitals. This is justified in the sense of innovation and integration into the labour market, which are standpoints virtually developed by the economic paradigm. It is noteworthy that the restricted humanistic capital in formal education has negatively resulted in the individuals' democratic political development, because this restriction inhibits political knowledge acquisition, which would shape the prerequisites for system interventions and monitoring (Nussbaum, 2013).

\section{Secret codes to compliance and discipline about teachers" "over- employment"}

The training courses market is continuously being expanded. A teachers' training model is gradually being shaped based on teachers' needs interpretation. Their time is monitored within a predetermined project ${ }^{3}$. The available working time is constantly being increased by the artificially supplementary and indirectly mandatory teachers' participation in training. Consequently, the time allocated to study, exploration or personal satisfaction is significantly being decreased. The imposed needs are conducive to generating new forms of teacher professional development resulting in an experienced condition of uncertainty and insecurity through shifting from one training to another, from one cognitive field to another. Finally, the concepts of useful time 
and knowledge are altered, as useful time is not separated from working time and knowledge is not separated from the defined useful professional one.

Teachers of different subjects purchase and consume or even become the inspirations of training courses. A multi-dimensional educational game is being unfolded, characterised by the combination of various educational courses, carriers and reconstructed educational reality. Each educational program is distinctive of its selective relations to any other program available in the market. Therefore, it utilizes any operational principles and standpoints towards its beneficial disposition. The training courses market has recently focused on the increasing number of potential teachers that is, unemployed teachers, creating, in this way, expectations for employment ${ }^{4}$. In this respect, the analytical categories of educational discourse are supplemented by themes pertaining to teachers' integration into the labour market based on the corresponding adapted interpretation of legislation.

The number of people attending training courses constantly rises. Educational institutes reinforce the provision of mainly brief educational courses, while the content is being less emphasized. This is so because focus is placed on inspiration and invention, while contemporary marketing terms are utilized along with instrumentally using social networks (Borgatti \& Everett, 2018; Christakis \& Fowler, 2011). In this sense, certifications, and communicative means are utilized towards attracting the "consumers'" interest (Baudrillard, 2016; Hovland \& Wolburg, 2014; Murphy, 2016).

Given this condition, teachers or potential teachers are continuously busy and tied to a process of accumulating even more degrees or certificates. It is all about participating in a developing game relevant to automatic procedures of nonconscious involvement in undefined and unclassified educational structures. Being involved with these courses is necessary for teachers in the form of a vague conceptualisation of assessment. On the other hand this is also necessary for potential teachers in the form of a vague interpretation of their qualifications as a means to integrate into the labour market. This pattern should also include the expanding group of career seekers who wish to be included in the bureaucratic authoritative mechanisms.

Along with accumulating undergraduate and postgraduate degrees as well as other conventional diplomas, certification acquisition is also on the rise. In this vein, through a vague approach to institutional process, amidst a legislative fragmentation and a downgraded condition of the educational function, multilevel educational training courses are being developed. Any serious theoretical approach is displaced while an obsessive quest for degrees and certifications is being unfolded. Time, in the sense of teachers or potential teachers' personal time is being shrunk within an adaptive functional, imposed settlement of "demands" and prerequisites.

Finally, teachers' leisure time (Kalerante, 2013) is at risk, resulting in a new form of reality without productive interest-related content and self-fulfilment due to selective knowledge. Confinement in the training courses process restricts, in political terms, individuals' self-determination and autonomy since the teachers' 
social self is being monitored within a distorted view about their role. In psychological terms, teachers are found to experiencing burn out syndrome which is the outcome of the broader social deregulation, their professional disdain and psychological enfeeblement. Consequently, the phenomenon of contemporary teachers' alienation (Hall, 2018) and isolation is on the rise, as this is the outcome of increasing rigid responsibilities. Both teachers' creativity and their functional relation to a broader content of essential knowledge towards shaping experiences within a unifying interdisciplinary view seem to be refuted.

A form of differentiation and isolation is developed in the educational environment, as attitudes and behaviours reinforcing individual courses to fulfil arbitrary or notional goals are being generalized. Confinement in individualcentred approaches is conducive to ruptured relations with collectivities; therefore, weakening the educational community ${ }^{5}$. Based on the capitalist rationale of predetermined needs which in turn are fragmented in individual arbitrary patterns, education faces a similar reality of teachers' adaptive upgrading in the form of individual value generalization due to insecurity, uncertainty and fluidity.

The teachers' adaptive upgrading model includes assumed individual choices to accumulating qualifications within a selectively interpreted modernization tied to adaptation. Disciplined individuals, involved in educational courses seem to converge in choices which are not actually theirs, but rather imposed. The secret working time on training courses is gradually being added to real working time. Different role patterns are being shaped putting forward labour and the capacity of the professional teacher at the expense of the right to self-expression, autonomy, reflection and personal interpretative understanding. Educational time and space are constantly being expanded, by associating with and internalising normative demands which eventually result in a dominant educational paradigm towards activating corresponding predispositions and monitoring behaviours. This is an example reflecting the political dysfunction of institutional carriers and corresponding structures.

It is well-understood that, through this individual consumerism of educational courses, the concept of collectivity and negotiation of meanings within the educational community is ruptured. A competition is indirectly being shaped, while individuals' marginalisation practices are being composed. Therefore, certain issues are put forward tied to the implementation of the policy of intentions relevant to schools evaluation, internal assessment of instruction and, more generally, to functionality and effectiveness of interactive processes in the educational environment.

\section{Re-designing functional educational courses or seeking educational empirical incidents}

Differentiating patterns that showcase citizens' society are put forward within the framework of conceptualised democratic re-adaptations and transcended political crisis. The educational community is emphasized on the micro-level of education. However, this is a notional process tied to a formal reference to the 
educational community aiming at achieving certain objectives. Although the educational environment is demonstrated in the form of an open system characterized by channels of communication and information input, it virtually operates as a cloze system in which balancing rational choices and the volunteering goal-setting of the educational community and education policy are refuted.

The criticism on the content of training courses does not downgrade the necessity to design such courses. The drawback of the training process is actually its fragmented space and involvement of different carriers without having established an explicit institutional framework. As a result, a chaotic condition is being developed, distinctive of deregulated practices, convergences and generalizations, within a vague status of optional, yet mandatory, training. The increasing number of training courses should not be interpreted in a singlesided manner that is, the result of "obsolete" university education. Moreover, vague reference to powerful educational carriers responsible to design training courses and the conscious invention of their necessity towards a condition of turning these courses into products and, in turn, teachers into consumers are not useful at all.

The institutional framework, real educational carriers and symbolic or nonsymbolic structures of the educational environment should all be emphasized. Political instruments and the broader educational community are all part of the broader educational environment. Therefore, any practices should be meaningful and not vague within the real content of the educational space that includes the political capital, scientific knowledge and democratic humanistic conceptualisation. The increasing number of training courses and institutes that do not belong to formal education (managers, etc.) involvement in them are gradually shifted to, perhaps, unreasonable symbolisation resulting from meanings and practices in education policy. To put it differently, training courses designing and delivery by educational institutes that do not belong to formal education should be revisited along with the practice of redesigning these courses (Diplari \& Dimou, 2010).

These uncontrollably developed educational institutes that do not belong to formal education should be monitored according to certain criteria so that they are gradually deconstructed. Essential communication with the educational community should be the focal point when designing new training courses. Highlighting symbolic democratic construction is of utmost importance. The distorted political legislative texts and corresponding policy practices should be pinpointed through expanded discourse fields in the educational community. Teachers' training should be redesigned on the basis of flexible courses combining theory and practice along with teachers' needs. Furthermore, a qualitative differentiation of training courses should seek pluralism in views, standpoints and considerations in tandem with teachers, unionists, university and research centres' democratic involvement.

Given that private training institutes take advantage of the lacking co-ordinated policy, their re-examination and assessment is necessary in order to ban rushed and fragmented courses solely based on profit. On the grounds of revisiting 
convergence policies about the cooperation among Primary and Secondary Education, Universities and research centres, a unifying policy towards reforming training courses could be established. This would derive from exploring educational issues within a functional re-association between scientific research centres and theoretical as well as implemented policy.

Besides, the transformed training courses, stemming from institutionalisation and internalised differentiated criteria on training, should be included in the new content of assessing teachers and selecting education executive personnel. Refuting or enfeebling non-operational courses can only derive from initiatives taken by scientific carriers and the educational community. Therefore, a readapted typology of training courses can gradually be developed based on education-related scientific discourse, directly consistent with institutional structures and broader educational interactive processes.

Knowledge searching is instrumentally tied to the transformed educational environment. Different views in the educational community must be coarticulated so that training courses are developed according to teachers' needs. Teachers' deliberation, juxtaposition or consent to the necessary knowledge can generate new fields of communication by defining control policies of educational processes held by teachers themselves. Amid a period in which conservative political structures wish to compose submissive forms of consciousness, deliberating could lead to productive educational discourse on the one hand and compose democratic interventions in the educational environment on the other.

\section{Concluding remarks}

Now is perhaps the most suitable time period for the educational community to liberate from the condition of accumulating degrees or certifications not based on scientific criteria or personal interests. Design and scientific production of knowledge should stem from teachers' needs, defined by teachers themselves, while distancing from predetermined interpretations. This change entails the transcendence of the formal process of qualifications acquisition. Dominant educational models tied to training courses consumption should be replaced by new tools that contribute to dynamic educational discourse construction. This translates to a critical approach to training courses offered, emphasizing on content and carrier, on the one hand and the reformed broader educational conceptual tools on the other. Thus, interest is shifted to educational action, the function of the educational community, views exchanging and reinforced communication among teachers, which are all factors expressing theoretical democratic composition.

The content of themes tied to internal and external educational functions, such as assessment and selecting education executives in corresponding bureaucratic structures should be redefined by the education policy. Uncertainty and insecurity are further intensified by the arbitrary content of assessment. The individuals' democratic co-operative policy, teachers in particular, has not obviously been put forward. Teachers must co-operate and interact towards 
maximizing the teaching work efficacy and their personal fulfilment as individuals, citizens and social subjects.

As regards the selection of individuals to staff executive positions in educational structures, a single-sided focus on the quantitative appraisal of qualifications is observed. Despite policy intentions, the selection of these executives is confined in obsolete patterns. As a result, juxtapositions about the political interpretative orientation of the educational organization are generated. In other words, a narrative discourse is constructed through secret codes which perhaps refute the policy of intentions for effective education executives' selection.

It seems that both the distorted assessment and education executives' selection process reinforce the "consumption" of training courses, as they simultaneously hold back reflection on a series of educational problems. Practices of redefining educational objectives and the teachers' role in the educational process are virtually rejected. Bearing in mind that training is necessary and that there are notable training courses, the democratic prolific dialogue should be the means by which to re-approach the relation between educational carriers and educational courses within a broader framework of educational culture and discourse.

Conceptual approaches, extricated from holistic views, should direct teachers to deliberation, juxtapositions, transformations, re-compositions and changes. The emerging challenge is to transcend passive consent through reinforced contradictory discourse. In this respect, a setting for democratic deliberation is formed by enhancing democratic principles and humanistic patterns (Traverso, 2014). At the same time, the teacher's social and political role is highlighted beyond professional capacity. Criticism on training courses eventually works as a process of transcending passive acceptance and leads to broader considerations on the teacher's role based on the historic deconstructive coincidence (Agamben et al., 2013; Graber \& Levinson, 2018; Kondiadis, Spyropoulos \& Tsoukalas, 2012). The educational community is invited to articulate anew democratic political discourse, while restricting the individual strategic estimations. Teachers are invited to re-approach the concept of community, within different disciplines and patterns, and juxtapositions to individual obsessive projection (Kitchen, 2014; Renaut, 2007).

\section{Recommendations}

The state should officially assume teachers' training in collaboration with the educational community. In this respect, new training courses could be developed to meet scientific terms, so that they are suitable for teachers, as they are part of the scientific community, too. Syndicalist unions and scientific associations should also be considered when developing teacher training courses, as they can evaluate the true needs of the educational community. Certificates provided by public carriers and private institutes that meet the requirements for their scientific work should be taken into consideration when evaluating education executives. On top of that, collaboration with university departments is deemed necessary, as scientific groups of various fields could 
participate in developing such training courses as well as teaching certain modules. All things considered, teachers' training should be supervised by the state and be part of the broader education policy, so that teachers' training is not a fragmented process.

\section{Notes}

${ }^{1}$ Throughout this period private initiative on all levels is highlighted as solution by showcasing all those features considered to be lacking in the public sector, such as meritocracy, transparency, opportunities structures about innovation and progress.

2 Each time reference is made to non-action of unionist carriers further reference is also made to the general political inertia, characteristic of political collectivities and fueling citizens' lack of trust to unionist carriers.

${ }^{3}$ Quote the movement actions being currently developed in France in which teachers, through internet communication, are being developed in pressure groups ("red pens") putting forward the educational community demand for invisible issues such as educational time not being assessed within educational work appraisal. This movement action is perhaps conducive to manifest transcendence of silence and rethinking of intellectuals' participation in policy in different terms. https:// www.connexionfrance.com/French-news/Stylos-rouges-teacher-protestmovement-grows-across-France-in-image-of-gilets-jaunes

Similar movement actions have been realized by American teachers who claim that quality in education is not achieved through schools assessment. Their movement actions are a means to highlight broader educational issues towards the gradual enfeeblement of public education, especially the right of non-privileged social classes to education. https://monde-diplomatique..gr/?p=3184

4 Along with educational knowledge market, a similar one of communicative skills is being developed focusing on shaping particularly unemployed individuals' attitudes and behaviors in order to achieve their integration into the labor market.

${ }^{5}$ See (indicatively) about recent researches on lacking trust to institutional political carriers and the decreased participation in collective carriers https://left.gr/news/ereyna-tis-public-issue-gia-tin-axiopistia-ton-thesmon-vathia-

dihasmeni-i-koinonia-apaitei kat https://kaparesearch.com/wp-
content/uploads/2017/12/KapaVima_Dec13_GR.pdf

\section{References}

Agamben, G., Badiou, A. \& Bensaid, D. et al., (2013). Where does democracy go? Athens: Patakis.

Baudrillard, J. (2016). The Consumer Society. London: Sage.

Belfield, C., \& Levin, H. (2007). The Price We Pay. USA: Brookings Institution Press.

Borgatti, S., \& Everett, M. (2018). Analyzing Social Networks. London: Sage.

Christakis, N., \& Fowler, J. (2011). Connected. New York: Back Bay Books.

Diplari, A., \& Dimou, E. (2010). Public tourism education and training in Greece: a study of the necessity for educational restructuring. Industry and Higher Education, 24(2): 115-120.

Graber, M., \& Levinson, S. (2018). Constitutional Democracy in Crisis. Oxford: Oxford University Press.

Graeber, D. (2018). Bullshit Jobs. New York: Simon \& Schuster. 
Hall, R. (2018). The Alienated Academic. USA: Palgrave Macmillan.

Harvey, D. (2007). Neoliberalism: History and Present. (A. Alavanou, transl.). Athens: Kastaniotis.

Hovland, R., \& Wolburg, J. (2014). Advertising, Society, and Consumer Culture. London: Routledge.

Kalerante, E. (2013). Greek Educational Policy in Recessionary Times: Does Liberal Educational Policy Take its Toll on the Leisure Activities of the Lower Social Strata? In F.M. Lo Verde, I. Modi \& G. Cappello (Eds.), Mapping Leisure Across Borders (379-389). Newcastle upon Tyne: Cambridge Scholars Publishing.

Kalerante, E. (2015). Political Ambiguities of Lifelong Learning: Between Humanism and Technocratism. In K.G. Karras, P. Calogiannakis, C.C. Wolhuter \& D. Kontogianni (Eds.), Education and Teacher Education in the Modern World: Problems and Challenges (253-267). Newcastle upon Tyne: Cambridge Scholars Publishing.

Kalerante, E. (2018). Determination of educational practices in market terms: Hybrid forms of post-secondary education as deliberating educational proposal. Public and Private Education, Proceedings of the $3^{\text {rd }}$ Panhellenic Conference of Sociology in Education (93-104). European University of Cyprus, Nicosia, 27 th and $28^{\text {th }}$ April 2018.

Kitchen, W. (2014). Authority and the Teacher. New York: Bloomsbury Academic.

Kondiadis, X., Spyropoulos, F. \& Tsoukalas, K. (eds.). (2012). Democracy, Constitution, Europe in the era of crisis. Athens: Savvalas.

Makrakis, V. (1997). Perceived relevance of information technology courses to prospective teacher' professional needs: the case of Greece. Journal of Information Technology for Teacher Education, 6(2): 157-167. Doi:10.1080/14759399700200014.

McMinn, D. (2015). Lifelong Learning. Cambridge: iPlace Press.

Murphy, W. (2016). Consumer Culture and Society. London: Sage.

Nussbaum, M. (2013). Not for profit. (G. Christidis, trnsl.). Athens: Kritiki.

Renaut, A. (2007). The end of mastery. (G. Karabelas, transl.). Athens: Polis.

Sengupta, E., \& Blessinger, P. (2018). Refugee Education: Integration and Acceptance of Refugees in Mainstream Society. United Kingdom: Emerald Publishing.

Traverso, E. (2014). What happened to the intellectuals? (N. Kourkoulos, transl.). Athens: Publications of the Eikosto Proto.

Zervas, Th. G. (n.d.). How Functional are Greek Teaching Programs? Teacher Training and Job Placement in Greece's Most Struggling Profession. Athens Journal of Mediterranean Studies, 2(4): 349-356. Doi:10.30958/ajms. 2-4-4. 